\title{
Spectroscopic survey of post-AGB star candidates ${ }^{\star}, \star \star$
}

\author{
C. B. Pereira ${ }^{1}$ and L. F. Miranda ${ }^{2}$ \\ 1 Observatório Nacional-MCT, Rua José Cristino 77, CEP 20921-400 São Cristóvão, Rio de Janeiro-RJ, Brazil \\ e-mail: claudio@on.br \\ 2 Instituto de Astrofísica de Andalucía, CSIC, Apdo. 3004, 18080 Granada, Spain \\ e-mail: lfm@iaa.es
}

Received 16 November 2005 / Accepted 19 September 2006

\section{ABSTRACT}

\begin{abstract}
Aims. Our goal is to establish the true nature of post-AGB star candidates and to identify new post-AGB stars. Methods. We used low resolution optical spectroscopy and we compared the spectra of the candidate post-AGB stars with those of stars in the library specta available in the literature and with spectra of "standard" post-AGB stars, and direct imaging in narrow-band filters.

Results. Spectra were obtained for 16 objects: 14 objects have not been observed previously and 2 objects are already known postAGB stars used as "standards" for identification. From the spectra we identify: six new post-AGB stars with spectral types between G5 and F5, two H II regions the morphology of which is revealed in the direct images for the first time, a G giant with infrared emission, a young stellar object, a probable post-AGB star with emission lines and three objects for which the classification is still unclear. As a whole, our results provide new, reliable identifications for 10 objects among listed post-AGB star candidates.
\end{abstract}

Key words. stars: AGB and post-AGB - H II regions - stars: pre-main sequence

\section{Introduction}

Post-AGB stars are luminous objects of low/intermediate mainsequence mass $\left(M_{\star} \leq 8 M_{\odot}\right)$ in the last stages of their evolution. They are the descendants of Asymptotic Giant Branch (AGB) stars and the immediate precursors of planetary nebulae (PNe). Post-AGB stars evolve toward higher effective temperatures, but they are not hot enough yet to ionise the surrounding remnant of the AGB envelope and to have entered in the PN phase. Therefore, these objects contain valuable information not only about the mass loss, chemical enrichment, and wind properties in the AGB phase, but also about the processes leading to the departure from AGB and those that will shape PNe (see Van Winckel 2003; and Engels 2005, for recent reviews). It is well known that evolution from the AGB to the PN phase involves dramatic changes in all stellar and circumstellar properties, for which many physical processes, still not well clarified, appear to be responsible (see Balick \& Frank 2002). Understanding the AGB to $\mathrm{PN}$ transition depends on precise knowledge of the formation and properties of post-AGB stars and how these objects evolve before they transform in PNe.

Even though new post-AGB stars have recently been identified (Suárez et al. 2006), the number of known post-AGB stars is still scarce (see also Szczerba et al. 2003). This is in part due to the short duration of this phase, which strongly depends on the initial mass. Although for the less massive objects the duration of

* Based on observations collected at the Centro Astronómico Hispano Alemán (CAHA) at Calar Alto, operated jointly by the MaxPlanck Institut für Astronomie and the Instituto de Astrofísica de Andalucía (CSIC), and at the Observatorio de Sierra Nevada, which is operated by the Consejo Superior de Investigaciones Científicas through the Instituto de Astrofísica de Andalucía (Granada, Spain).

$\star \star$ Appendices A-D are only available in electronic form at http://www. aanda.org the post-AGB phase may be as large as $10^{4}-10^{5} \mathrm{yr}$, more massive objects may last only a few decades/centuries in the postAGB phase (Blöker 1995). In these circumstances, identification of post-AGB stars may be difficult. The IRAS satellite was successful in enabling systematic identifications of post-AGB candidates. The IRAS [12]-[25] versus [25]-[60] colour-colour diagram and the $J-K$ versus $H-K$ diagram (Preite-Martínez 1988; García-Lario et al. 1997, hereafter GL97) have been widely used to identify a large number of possible objects in the post-AGB phase. However, these diagrams alone do not allow us to clearly distinguish between post-AGB stars and other kind of objects (e.g., H II regions, young stellar objects, AGB stars). To determine the true nature of the candidates, other observations are necessary. The purpose of this paper is to present and discuss the spectra of several candidates to post-AGB stars, which have been obtained to establish their true nature and, thereby, to identify new post-AGB stars.

\section{Selection criteria}

The objects were selected for observation in the framework of our low-resolution spectroscopic study of a large sample of Preite-Martínez's (1988), PN candidates as well as from GL97 transition objects (AGB and post-AGB stars to proto-PNe and $\mathrm{PNe}$ ). To select our objects we follow the same procedure of Pottasch et al. (1998) which was also used by Preite-Martínez (1988) to select and distinguish PNe. However, we used different lower and upper limits in IRAS colours in our selection following GL97. In brief, if a post-AGB or a PN candidate fulfills a criterion of having IRAS flux ratios such as $F(12 \mu \mathrm{m}) / F(25 \mu \mathrm{m}) \leq$ 0.50 and $F(25 \mu \mathrm{m}) / F(60 \mu \mathrm{m}) \geq 0.35$, then there will be a good chance for it being either a true post-AGB star or a true PN. In the IRAS colour-colour diagram, the IRAS flux ratios set a 
Table 1. List of objects spectroscopically observed.

\begin{tabular}{lccccc}
\hline \hline Star & IRAS & {$[12]-[25]$} & {$[25]-[60]$} & & $b$ \\
\hline PM 1-212 & $18044-1303$ & $\leq+2.15$ & +0.81 & 1 & +3.6 \\
PM 1-243 & $18321-1401$ & $\leq+1.36$ & +0.53 & 2 & -2.8 \\
PM 1-261 & $18489-0629$ & $\leq+3.05$ & +0.59 & 3 & -2.9 \\
PM 1-267 & $18539+0549$ & $\leq+2.68$ & +1.07 & 4 & +1.6 \\
PM 1-312 & $19422+1438$ & $\leq+2.09$ & -0.03 & 5 & -4.7 \\
GLMP 9 & $00422+6131$ & +0.96 & +0.89 & 6 & -1.12 \\
GLMP 75 & $04302+4425$ & +0.90 & +0.34 & 7 & -2.2 \\
GLMP 818 & $18386-1253$ & +2.13 & +0.04 & 8 & -3.7 \\
GLMP 982 & $20136+1309$ & +0.88 & -1.67 & 9 & -11.9 \\
GLMP 1000 & $20324+4057$ & +1.01 & +0.79 & 10 & +0.5 \\
GLMP 1015 & $20572+4919$ & +1.01 & -0.29 & 11 & +2.4 \\
GLMP 1043 & $21525+5643$ & +1.07 & -0.29 & 12 & +2.0 \\
GLMP 1058 & $22223+4327$ & +3.11 & -0.55 & 13 & -11.6 \\
GLMP 1062 & $22331+5809$ & +2.36 & +1.01 & 14 & +0.1 \\
GLMP 1078 & $23304+6147$ & +1.79 & -0.87 & 15 & +0.6 \\
GLMP 1083 & $23436+6306$ & +0.88 & +0.92 & 16 & +1.4 \\
\hline
\end{tabular}

lower and an upper limit as [12]-[25] $\geq 0.75$ and [25]-[60] $\leq$ 1.13 , respectively, according to

$$
\begin{aligned}
& {[12]-[25]=-2.5 \log \frac{F_{12 \mu \mathrm{m}}}{F_{25 \mu \mathrm{m}}}} \\
& {[25]-[60]=-2.5 \log \frac{F_{25 \mu \mathrm{m}}}{F_{60 \mu \mathrm{m}}}}
\end{aligned}
$$

This criterion was used by GL97 as an additional constraint, besides near infrared photometry, to determine the nature and the evolutionary status of the objects in their sample. Post-AGB stars and $\mathrm{PNe}$ following this criterion would be inside a box defined by the colours mentioned above. However, young stellar objects, $\mathrm{H}$ II regions, or variable $\mathrm{OH} / \mathrm{IR}$ stars also fall inside this box, so that additional observations are necessary to discriminate the true nature of the objects. Since our main goal is to find new post-AGB stars, we include two well known post-AGB stars in our spectroscopic survey as "standard post-AGBs": GLMP 1058 and GLMP 1078 (van Winckel \& Reyners 2000). In addition, we include GLMP 1015 in our list, which has already been claimed as post-AGB star (Hrivnak et al. 1994; Arkhipova et al. 2000), but has never been spectrocopically observed before. Table 1 gives the object's name from GL97 and Preite-Martínez (1988), the IRAS name, the IRAS colours, a sequence number (see below), and the galactic latitude.

Following GL97, in Fig. 1, we show the position of the spectroscopically observed objects in the IRAS two-colour diagram, according to the sequence number in Table 1 . We also include in Fig. 1 a new, identified young PN, PM 1-322 (No. 17) (Pereira \& Miranda 2005), and the already known post-AGB star GLMP 160 (No. 18) (Pereira \& Machado 2003). We note that for PM 1-243, PM 1-261, PM 1-267, and PM 1-312, the $12 \mu \mathrm{m}$ IRAS fluxes are upper limits. Therefore, their positions in the axis [12]-[25] are also upper limits. The solid line in Fig. 1 is the exponential curve calculated by Bedijn (1987) for the change of IRAS colours for an AGB star with increasing mass loss. Figure 1 also shows four boxes that are associated with : a) Mira variable; b) variable $\mathrm{OH} / \mathrm{IR}$ star; c) young stellar object; and d) compact H II regions (see GL97). There are 7 objects outside these boxes, while they follow the criteria given by their IRAS colours to be a post-AGB star. Two of them are the "standard post-AGBs" GLMP 1058 (No. 13) and GLMP 1078 (No. 15), and a third one is the post-AGB star GLMP 160 (No. 18). The objects PM 1-261 (No. 3), PM 1-312 (No. 5), GLMP 818 (No. 8),

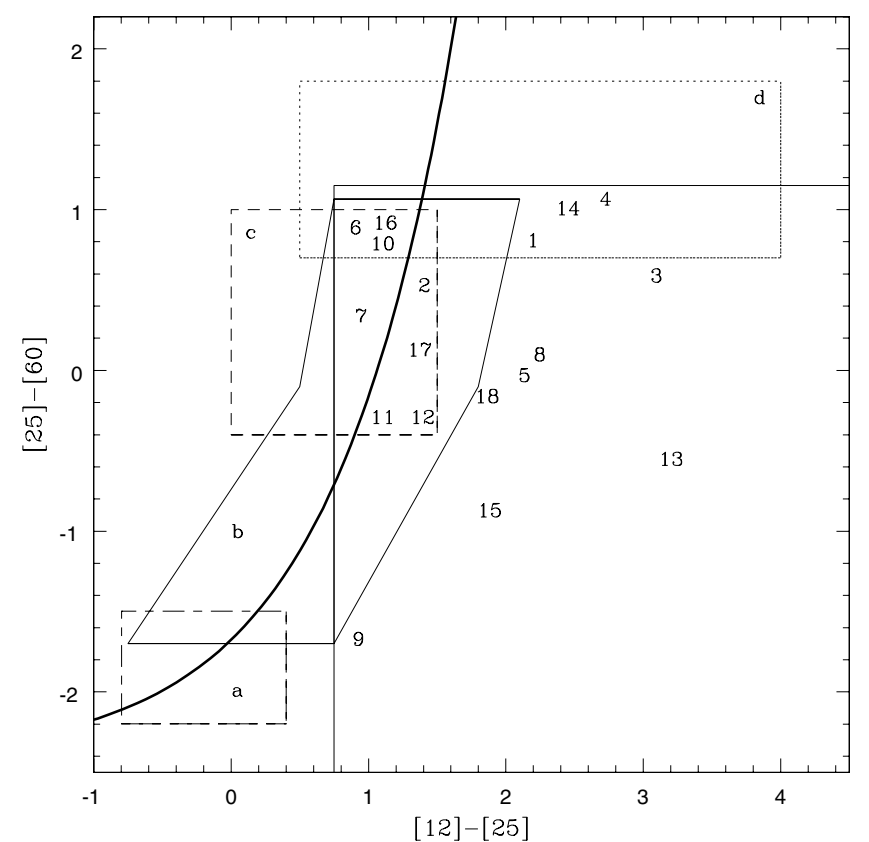

Fig. 1. The position of the spectroscopically observed objects in the IRAS two-colour diagram. See Table 1 for the corresponding names. We also give the positions of two previous analysed objects. 17: PM 1322 (Pereira \& Miranda 2005) and 18: GLMP 160 (Pereira \& Machado 2003). The boxes labeled a, b, c, and d define the regions populated by Mira variables, variable $\mathrm{OH} / \mathrm{IR}$ stars, young stellar objects, and compact $\mathrm{H}$ II regions, respectively.

and GLMP 982 (No. 9) should be considered post-AGB stars. The rest of the objects may be post-AGB stars (including PNe, e.g., PM 1-322 No. 17), young stellar objects, or compact H II regions since their IRAS colours overlap. We note that narrowband images have been obtained for four of the objects observed spectroscopically. Because the images provide new information about these four objects, we include them in this work.

\section{Observations and reductions}

Spectroscopic observations were performed with CAFOS (Calar Alto Faint Object Spectrograph) attached to the $2.2 \mathrm{~m}$ telescope at the Calar Alto Observatory (Almería, Spain) during August 24-26, 2004 and December 1, 2004 and August 3-7, 2005 The detector was a SITe CCD with 2048 pixels in the spectral direction. The observations were done using the grisms B100 and R-100 covering the spectral range $3200-6000 \AA$ and 5900-9300 ̊, respectively (for GLMP 75 only the range 3200 $6000 \AA$ was observed, while for GLMP 1000 we observed only the range 5900-9300 $\AA$ ). Exposure time ranges between $600 \mathrm{~s}$ and $3000 \mathrm{~s}$, depending on the brightness of the object. The resolution obtained with both grisms is $2 \AA / p i x e l$ and the slit orientation was north-south. Spectrophotometric standards from Oke (1974) and Massey et al. (1988) were also observed for flux calibration. The spectra were reduced using standard IRAF tasks.

Direct images of post-AGB candidates were obtained during August 3-15, 2004 with the $1.5 \mathrm{~m}$ telescope at the Observatorio de Sierra Nevada (Granada, Spain). The detector was a RoperScientific VersArray with $2048 \times 2048$ pixels of 0 '232 in size, resulting in a field of view of 7.9 $\times 7$ !.9. Images 
were obtained through three narrow-band filters: $\mathrm{H} \alpha\left(\lambda_{\mathrm{c}}=\right.$ $6563 \AA, F W H M=10 \AA),[\mathrm{N} \mathrm{II}]\left(\lambda_{\mathrm{c}}=6584 \AA, F W H M=10 \AA\right)$, and [O III] $\left(\lambda_{\mathrm{c}}=5007 \AA, F W H M=50 \AA\right)$. Exposure time was $1800 \mathrm{~s}$ for each filter. The spatial resolution, mainly depending on the seeing, varies between 1'.3 and 2'. 1 . The images were reduced following standard procedures within the MIDAS package. The imaged objects in common with the spectroscopic survey are GLMP 1000, GLMP 1062, GLMP 1083, and GLMP 9.

\section{Results and discussion}

In this section we will concentrate on the description of the spectra of the post-AGB stars in our sample, leaving the description of the data and identificacion of the rest of the objects for the section Online material (see also Sect. 4.3).

\subsection{New post-AGB stars}

\subsubsection{Identification}

From the observed candidate post-AGB stars, PM 1-243, PM 1261, PM 1-267, PM 1-312, GLMP 818, and GLMP 982 show spectral characteristics that allow us to identify them as new post-AGB stars. In the following we will provide evidence based on the spectroscopic data that these stars are of luminosity class I, which is one of the criteria for a star to be a post-AGB star (Kwok 1993). For this, we will compare the strengths of some suitable absorption lines in our candidate stars with those of stars from the library spectra available in the literature (Jacoby et al. 1984; Torres-Dagen \& Weaver 1993; Danks \& Dennefeld 1992). Finally, we will compare our candidate stars, whenever possible, with the two "standard post-AGB stars" GLMP 1058 and GLMP 1078 to better constrain their nature.

We have measured the strength (equivalent width) of the Paschen line at $8751 \AA$, the K line of Ca II at $3933 \AA$, the calcium infrared triplet, the oxygen line at $7774 \AA$, and the $\mathrm{CN}$ band at $7925 \AA$ in the spectra of the stars from Jacoby et al. (1984) and Danks \& Dennefeld (1992), in our six candidate stars and in the two standard post-AGB stars. Of the lines mentioned above, the most useful ones to distinguish supergiants from giants and dwarfs are the oxygen line at $7774 \AA$ and the CN band at $7925 \AA$. Figure 2 shows how the strength of the oxygen line changes according to the luminosity class. In dwarf stars the strength never reaches a value greater than $0.8 \AA$, in giants it is always less than $1.0 \AA$, while in supergiants it can reach values greater than $2.0 \AA$. In our candidate stars, this line is present, except in PM 1-267 (see also below), with equivalent widths of $2.0 \AA$, 1.9, $1.7 \AA$, $1.4 \AA$, and $1.4 \AA$ in PM 1-261, PM 1-312, GLMP 818, PM 1243 , and GLMP 982, respectively. In the two standard post-AGB stars, GLMP 1058 and GLMP 1078, the strength of the oxygen line is $1.7 \AA$ and $1.8 \AA$, respectively. In Fig. 2 we also show the position of our post-AGB stars. The spectral type assigned to them is approximate and based on a comparison with the spectra of the standard post-AGB and other standard stars (see below). Based on the temperature data available (van Winckel \& Reyniers 2000), we also plot the positions of the two standard post-AGB stars in this diagram. Even though the spectral types may be somewhat uncertain, the large equivalent widths of the oxygen line at $7774 \AA$ provide conclusive evidence that five of these stars are supergiants.

As for the $\mathrm{CN}$ band at $7925 \AA$, we notice that it has its greatest strength in supergiants with values up to $10 \AA$ for late $\mathrm{G}$ supergiants. In dwarfs it is never stronger (when present)

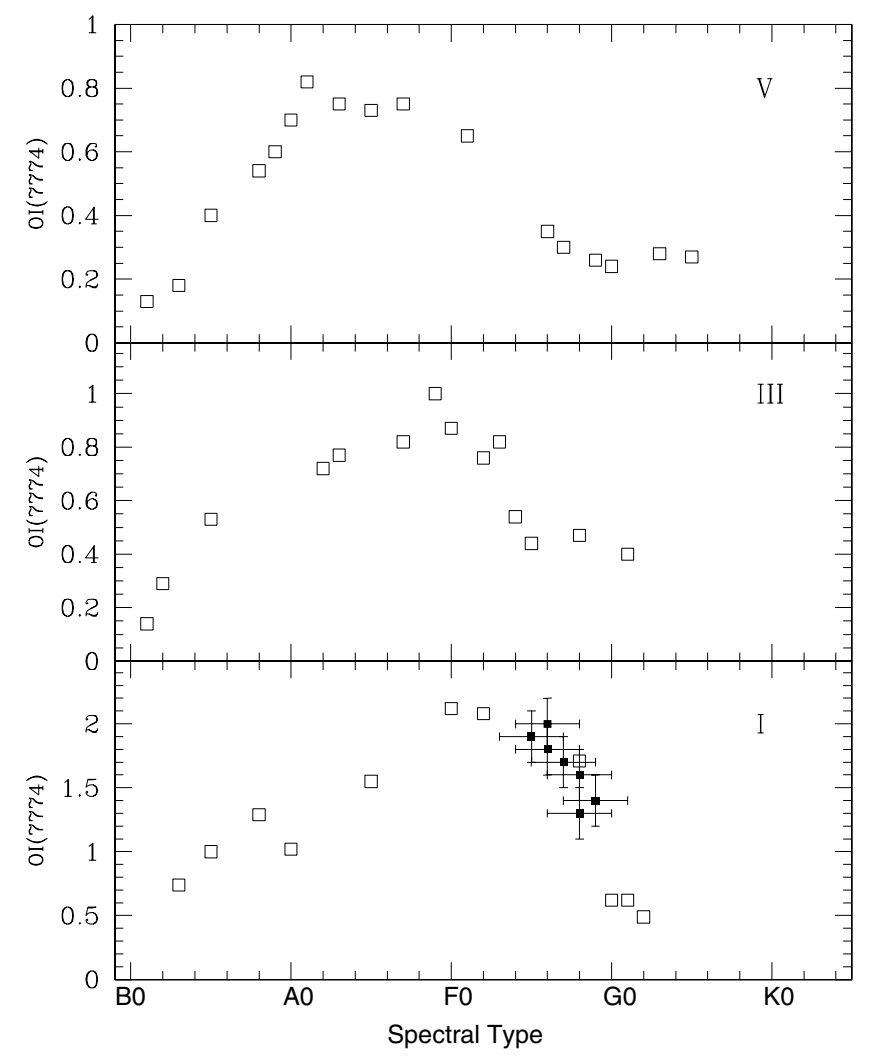

Fig. 2. The equivalent width $\AA$ of the O I $7774 \AA$ line versus spectral type for dwarfs, giants, and supergiants (open squares), labeled from top to bottom, respectively, as roman numerals as V, III, and I. Notice the strength of this line in supergiants as compared with giant and dwarfs. We also show the positions of the PM 1-261, PM 1-312, GLMP 982, GLMP 818, PM 1-243, GLMP 1058, and GLMP 1078 (filled squares).

than $1.4 \AA$ in the spectral range from $\mathrm{F} 6 \mathrm{~V}$ to $\mathrm{K} 0 \mathrm{~V}$. In giants, this strength lies between $0.7 \AA$ and $4.3 \AA$ in the spectral range F8III to K3III. The CN band is definitely present in PM 1-261, GLMP 818, and PM 1-267 with a strength of $2.4 \AA, 1.7 \AA$, and 2.6 $\AA$, respectively. In GLMP 1058 and GLMP 1078 it has values of $2.5 \AA$ and $2.4 \AA$, respectively. Its absence in PM 1-312, PM 1243 , and GLMP 982 probably indicates that these stars have a spectral type earlier than F6.

The calcium infrared triplet is a class luminosity discriminator, but only for stars beyond spectral type F5, since for earlier types it is severely blended with the Paschen lines (Danks $\&$ Dennefeld 1994). The calcium triplet reaches its strongest strength in the supergiants, from $10 \AA$ to $13.0 \AA$. In dwarfs and giants this value lies around $6 \AA$ and $8 \AA$, respectively (Jones et al. 1984). For our candidate stars, the strength of the calcium infrared triplet is $13.7 \AA, 12.6 \AA, 16.0 \AA, 9.0 \AA, 12.0 \AA$, and $9.0 \AA$ for PM 1-261, PM 1-312, GLMP 818, PM 1-267, PM 1-243 and GLMP 982, respectively. In the two standard post-AGB stars, GLMP 1058 and GLMP 1078, the values are $11.6 \AA$ and $10.7 \AA$, respectively.

The other two features, the Paschen line at $8751 \AA$ and the Ca II line at $3933 \AA$, were not useful to classify the post-AGB star candidates. Based on the Paschen line alone, we could not distinguish a giant from a supergiant. Our measurements of the Ca II line seem to indicate that it reaches its greatest strength in giant stars. However, the values for stars of spectral type later 


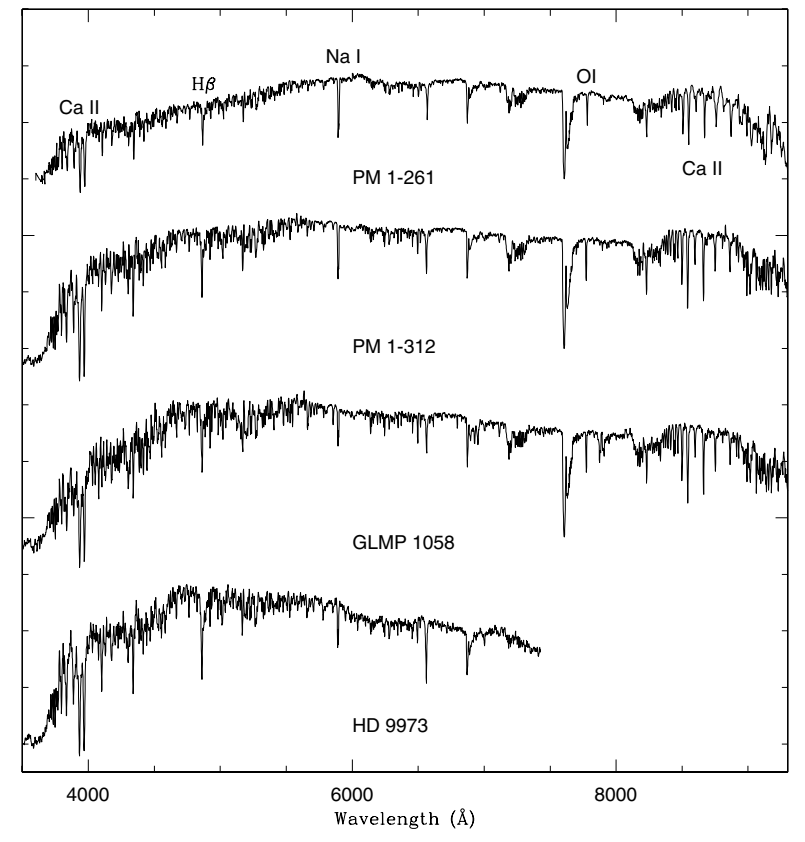

Fig. 3. Relative flux versus wavelength $(\AA)$ spectra of stars from a sample of Preite-Martinez (1988) and GL97 and the post-AGB star GLMP 1058. We also include a F5Iab star (HD 9973) reddened by $E(B-V)=0.5$ for comparison.

than F0 should be seen with caution because it is very difficult to set the continuum around this line in low resolution spectra.

From these measurements, we conclude that PM 1-243, PM 1-261, PM 1-267, PM 1-312, GLMP 818, and GLMP 982 are supergiant stars. This result, together with the IRAS colours, provides conclusive evidence that these six post-AGB candidates are, in fact, post-AGB stars. In the following we will comment about other spectral characteristics of these stars, which are also in consonancy with their classification as post-AGB stars.

The absorption spectrum of PM 1-261 and PM 1-312 is similar to that of GLMP 1058 (see Fig. 3) which has an effective temperature of $6500 \mathrm{~K}$ (van Winckel \& Reyniers 2000). Figure 3 also shows the spectrum of a typical F5Iab star (HD 9973) from Jacoby et al. (1984) reddened by $E(B-V)=0.5$. All these three stars present very strong $\mathrm{H}$ and $\mathrm{K}$ Ca II lines in absorption, which are typical of F-type star (Jacoby et al. 1984). The presence of the CN band in PM 1-261 and its absence in PM 1312 (see above) probably indicates that PM 1-312 is hotter than PM 1-261.

The continuum of PM 1-267 and GLMP 818 indicates very reddened stars (Fig. 4). In fact, using the library of stellar spectra by Jacoby et al. (1984), their continuum distribution can be better fit by a reddened F5I star. Figure 4 shows that the spectra are similar to that of GLMP 1078, which has an effective temperature of $6750 \mathrm{~K}$ (van Winckel \& Reyniers 2000). In addition, the Paschen lines are absent in PM 1-267. We also noticed that the absence of the oxygen line at $7774 \AA$ and the presence of the calcium infrared triplet indicate that PM 1-267 has a spectral type similar to that of a G5I star. In fact, a G5I star has a temperature of $4850 \mathrm{~K}$ (Schmidt-Kaler 1982), which is much lower than that of GLMP 1078.

It should be noted that PM 1-267 was classified as a PN by van de Steene \& Pottasch (1995) because of its radio continuum emission at $6 \mathrm{~cm}$. However, our spectrum does not show any emission line. A similar situation is observed in the Egg Nebula that does not show nebular emission lines, but strong radio

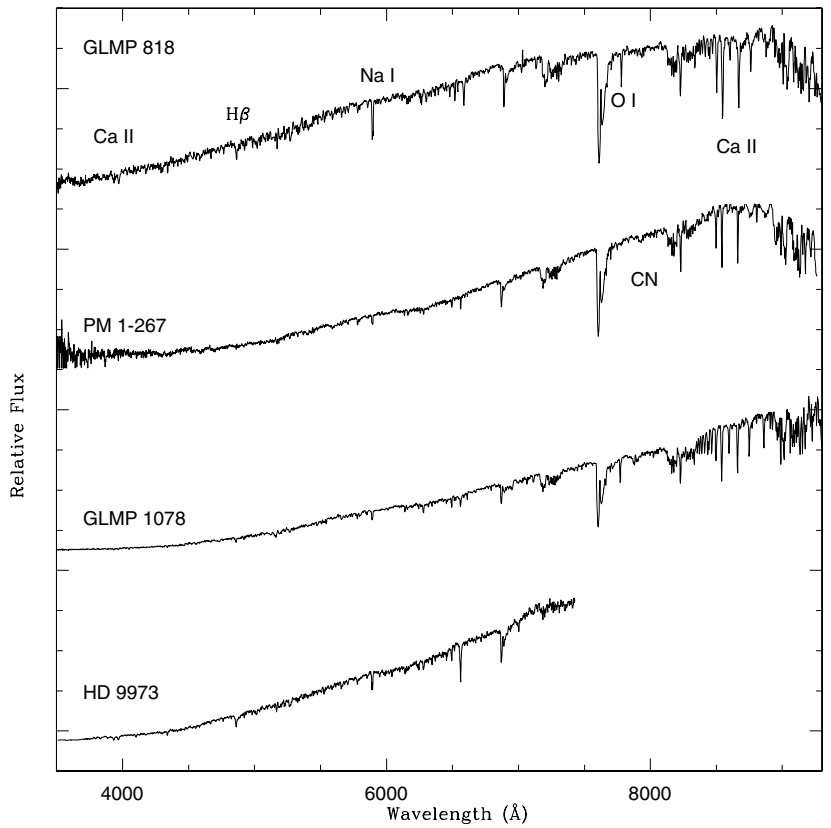

Fig. 4. Relative flux versus wavelength $(\AA)$ spectra of stars from a sample of Preite-Martinez (1988) and GL97 and the post-AGB star GLMP 1078. We also include a F5Iab star (HD 9973) reddened by $E(B-V)=2.0$ for comparison.

continuum emission at centimeter wavelengths, which is due to dustas indicated by the spectral index (Jura et al. 2000). Radio continuumn emission from PM 1-267 has been observed at only one frequency (van de Steene \& Pottasch 1995), and the spectral index cannot be calculated. Multi-frequency observations of the radio continuum emission from PM 1-267 will certainly allow us to deduce the origin of this emission.

PM 1-243 and GLMP 982 have a spectral type similar to that of a F-type star (Fig. 5), albeit with a lesser degree of reddening than the objects shown in Fig. 4, since the $\mathrm{H}$ and $\mathrm{K}$ lines of Ca II are observed. These two stars exhibit the same features as those observed in the F-type stars mentioned above such as the calcium infrared triplet and the oxygen triplet at $7774 \AA$. GLMP 982 is an oxygen-rich star, since no feature at $21 \mu \mathrm{m}$ was detected (Kwok et al. 1995).

\subsubsection{Search for signatures of s-process nucleosynthesis}

Post-AGB stars may present s-process enhancements. Although our spectra are of low resolution and, hence, not entirely suitable for detailed line identification, we carry out a search for absorption lines in the spectra of the post-AGB stars to probe the presence of elements created by slow neutron capture reactions. These lines include Ba II at $4554 \AA$, $5853 \AA$, $6141 \AA$, and $6496 \AA$, and Sr II at $4077 \AA$ and $4215 \AA$. In addition, we also inspect whether the carbon lines around $7110 \AA$ are present. We use GLMP 1058 and GLMP 1078 as template stars for this search since these two stars are already known to exhibit both carbon and barium enhancements (van Winckel \& Reyners 2000). We also used the library spectra of Barnbaum et al. (1996) and Allen $\&$ Strom (1995) for comparison. The first one is very useful because there are several peculiar stars ( $\mathrm{CH}$ and Barium) observed at almost the same resolution as ours and the later one because they present several spectra between $5700 \AA$ and $9000 \AA$, where one could detect, if present, C I around $7110 \AA$. 


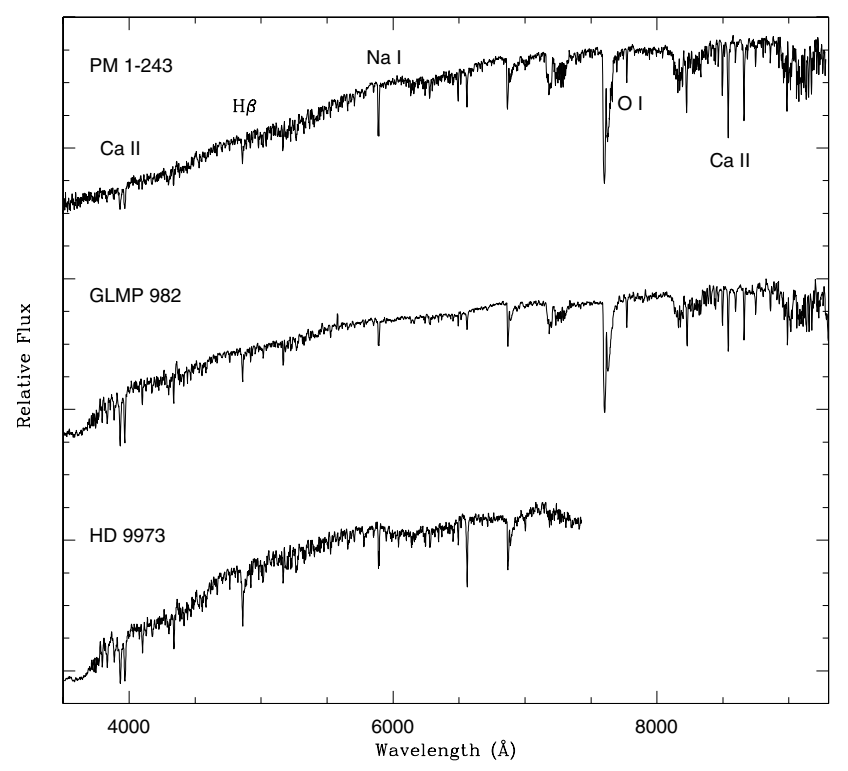

Fig. 5. Relative flux versus wavelength $(\AA)$ spectra of stars from a sample of Preite-Martinez (1988) and GL97. We also include a F5Iab star (HD 9973) reddened by $E(B-V)=1.0$ for comparison.

We were not able to distinguish the Ba II $4554 \AA$ and Sr II $4215 \AA$ lines in any of the stars among the several absorption lines in this region. The SrII $4077 \AA$ line is detected in GLMP 1058, GLMP 1078, PM 1-261, PM 1-312, and probably in GLMP 818. The Ba II lines at $5853 \AA, 6141 \AA$, and $6496 \AA$ are present in the spectra of the stars where the Sr II $4077 \AA$ line is detected. However, these three Ba II lines may not be useful, at the resolution of our data, to probe whether s-process nucleosynthesis has taken place because they are severely blended with other strong lines, such as Ca I $5857 \AA$, Fe I 6141 A, Ca I $6494 \AA$, Fe I $6495 \AA$, and Fe I $6496 \AA$. Perhaps the most promising feature to probe carbon enhancements is the carbon line around $7110 \AA$, since this line is apparently free of contaminations. We found a strong feature at $7110 \AA$ in GLMP 1058, GLMP 1078, PM 1261, PM 1-243, PM 1-312, and GLMP 818. The strength of this line is always stronger in the post-AGB stars than in A-F type standard stars used for comparison. We did not detect the carbon lines in GLMP 982 because it is an oxygen-rich star (see above) and in PM 1-267 because it is probably not hot enough to excitate this line. From these results we conclude that the stars with carbon and strontium features are good candidates to be investigated at higher resolution to determine their abundance pattern.

\subsection{GLMP 1015, a previously known post-AGB star}

GLMP 1015 is also known as VES 351 and V 2324 Cyg and, as already mentioned, has been considered as a post-AGB star (see Sect. 2). Our spectrum (Fig. 6) shows $\mathrm{H} \alpha$, the sodium doublet, O I $7774 \AA$, and O I $8446 \AA$ in emission. The calcium triplet is also in emission, which is a strong characteristic of young stellar objects (Hamann 1992a,b) and some symbiotic stars (Munari \& Zwitter 2002). Classified as F-type star by Hrivnak et al. (1994), our data shows the $\mathrm{H}$ and $\mathrm{K}$ lines of Ca II in absorption. The spectral type as well as the luminosity class of this star seem to be variable. Arkhipova \& Ikonnikova (1994) classified it as a B8-B9 II star and mentioned that GLMP 15 cannot be in the main sequence because this would imply a very high reddening. Later on, Arkhipova et al. (2000), based on broadband

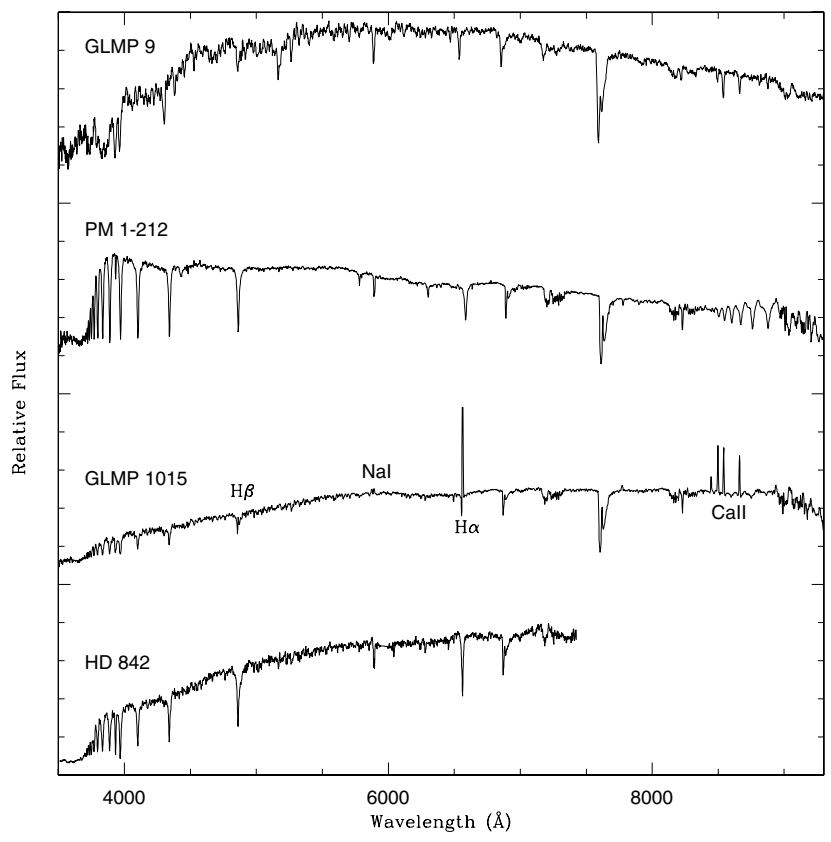

Fig. 6. Relative flux versus wavelength $(\AA)$ spectra of GLMP 9, PM 1212, GLMP 1015, and a A9I star (HD 842) reddened by $E(B-V)=1.0$.

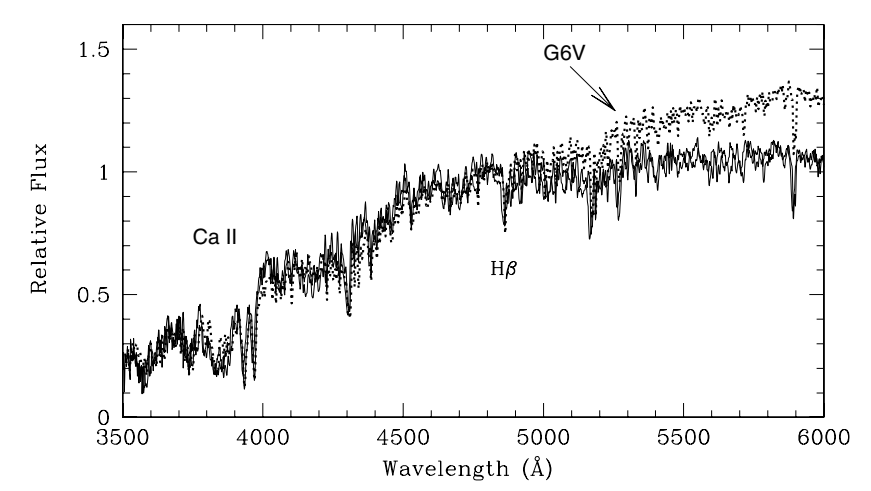

Fig. 7. Relative flux versus wavelength $(\AA)$ spectrum of GLMP 9 (solid line), a G7III star (solid line), and a G6V star (dotted line) reddened by $E(B-V)=0.5$. We see that the spectrum of GLMP 9 is very similar to the spectrum of a G7III star.

photometry, classified GLMP 1015 as a A3 I star. By comparing the $\mathrm{H} \delta$ measurements in our spectrum with the standards from Jacoby et al. (1984), it seems that GLMP 1015 has a spectral type of a late A-early $F$ supergiant star. Figure 6 also shows an A9I star reddened by $E(B-V)=1.0$ for comparison. It is interesting to note that even in the most recent papers, the supergiant nature of this star (if this is the case) is still not revealed either with infrared spectroscopy (Kelly \& Hrivnak 2005) or submilimiter observations (Hrivnak \& Bieging 2005). According to García-Lario et al. (1990) "an optical spectrum is necessary to confirm the nature of this object". However, from our data we cannot conclude whether this star is a supergiant since the absorption features used to provide a diagnostic of the luminosity class (e.g., the oxygen line at $7774 \AA$, see above) are in emission.

\subsection{Other post-AGB candidates}

The rest of the objects in Table 1 include two H II regions (GLMP 1000 and GLMP 1062), a young stellar object (PM 1212), a G giant with infrared emission (GLMP9), and three 
objects for which no classification was possible (GLMP75, GLMP 1043, and GLMP 1083). The data of these objects are presented and discussed in Appendices A to D (see Online material).

\section{Conclusions}

We have carried out a spectroscopic survey of post-AGB star candidates with the aim of establishing their true nature and of identifying new post-AGB stars. Narrow-band images have also been obtained for four post-AGB candidates not previously observed. The main conclusions of this work can be summarised as follows:

1) Six new post-AGB stars have been identified by comparing their spectra with spectra of stars with different spectral types and luminosity classes, and with those of already known post-AGB stars also observed in our survey. The new post-AGB stars are: PM 1-243, PM 1-261, PM 1-267, PM 1312, GLMP 818, and GLMP 982. Their spectral types range from G5 to F5.

2) GLMP 1000 and GLMP 1062, previously known as emission-line objects, are identified as $\mathrm{H}$ II regions because of their emission line ratios and morphology in the direct images. In GLMP 1000, a group of three nebulous objects is detected with a bow-shock or a disc/outflow appearance, while a complex region dominated by a large emission bow is found in GLMP 1062.

3) GLMP 9 is identified as a giant star (luminosity class III) rather than as a dwarf. The presence of infrared emission in GLMP 9 deserves further investigation.

4) PM 1-212 is identified as main sequence A-type star.

5) GLMP 1015 is probably a post-AGB star, although high resolution spectroscopy would be necessary to confirm its nature.

6) For GLMP 75, GLMP 1043, and GLMP 1083, no definitive classification could be done. Although the spectra suggest that they may be young stellar objects, in the case of GLMP 1083 the possibility of being a post-AGB star cannot be ruled out.

Acknowledgements. We thank our referee (H. Van Winckel) for detailed comments that have improved the presentation and interpretation of the data. We are very grateful to the staffs of the Calar Alto and Sierra Nevada Observatories for assistance during the observations. This work has been supported partially by grants AYA2002-00376 and AYA2005-01495 of the Spanish MEC (co-funded by FEDER funds). L. F. M. is also supported by Junta de Andalucía. This research has made use of the SIMBAD database, operated at CDS, Strasbourg.

\section{References}

Allen, L. E., \& Strom, K. M. 1995, AJ, 109, 1379

Arkhipova, V. P., Ikonnokova, N. P. 1994, Ast L, 20, 603

Arkhipova, V. P., Burlak, M. A., \& Esipov, V.F. 2000, Ast L, 26, 372

Balick, B., \& Frank, A. 2002, ARA\&A, 40, 439

Barnbaum, C., Stone, R. P. S., \& Keenan, P.C. 1996, ApJS, 105, 419

Bedijn, P. J. 1987, A\&A, 186, 136

Comerón, F., Pasquali, A., Rodighiero, G. 2002, A\&A, 389, 874

Danks, A. C., \& Dennefeld, M., 1994, PASP, 106, 382

Engels, D. 2005, Mem. S. A. It., 76, 441

García-Lario, P., Manchado, P., Pottasch, S. R., Suso, J., \& Olling, R. 1990, A\&AS, 82, 497

García-Lario, P., Manchado, P., Pych, W., \& Pottasch, S. R. 1997, A\&AS, 126, 479 (GL97)

Hamann, F. \& Persson, S. E. 1992a, ApJS, 82, 247

Hamann, F., \& Persson, S. E. 1992b, ApJS, 82, 285

Hrivnak, B. J., \& Bieging, J. H. 2005, ApJ, 623, 331

Hrivnak, B. J., Kwok, S., \& Geballe, T. R. 1994, ApJ, 420, 783

Jacoby, G. H., Hunter, D. A., \& Christian, C. A. 1984, ApJS, 56, 257

Jones, J. E., Alloin, D. M., \& Jones, B. J. T. 1984, ApJ, 283, 457

Jura, M. 1999, ApJ, 515, 706

Jura, M., Turner, J. L., Van Dyk, S., Knapp, G. R. 2000, ApJ, 528, L105

Kelly, D. M., \& Hrivnak, B. J. 2005, ApJ, 629, 1040

Kwok, S. 1993, ARA\&A, 31, 63

Kwok, S., Hrivnak, B. J., \& Geballe, T. R. 1995, ApJ, 454, 394

Lattanzi, M. G., Massone, G., \& Munari, U. 1991, AJ, 102, 177

Magakian, T. Yu. 2003, A\&A, 399, 141

Massey, P., Strobel, K., Barnes, J. V., \& Anderson, E. 1988, ApJ, 328, 215

Munari, U., \& Zwitter, T. 2002, A\&A, 383, 188

Pereira, C. B., \& Machado, M. A. D. 2003, A\&A, 407, 311

Pereira, C. B., \& Miranda, L. F. 2005, A\&A, 433, 579

Pottasch, S. R., Bignell, C., Olling, R., \& Zilstra, A. A. 1998, A\&A, 205, 248

Preite-Martinez, A. 1988, A\&AS, 76, 317

Oke, J.B. 1974, ApJS, 27, 21

Sabbadin, F., Minello, S., \& Bianchini, A. 1977, A\&A, 60, 147

Schmidt-Kaler, T. 1982, in Landolt-Börnstein New Series, ed. Schaifers, K., \& Vigt, H.H., Group 4, Vol. 2b (Berlin: Springer), 449

Suárez, O., et al., 2006, A\&A, in press

Szczerba, R., Górny, S. K., \& Zalfresso-Jundzillo, M. 2001, in Post-AGB objects as a phase of stellar evolution, ed. Szczerba, R., \& Górny, S. K., Astrophysics and Space Science Library, 265, 13

Torres-Dagen, A. V., \& Weaver, W. B., 1993, PASP, 105, 693

Van de Steene, G. C., \& Pottasch, S. R. 1995, A\&A, 299, 238

Van Winckel, H. 2003, ARA\&A, 41, 391

Van Winckel, H., \& Reyniers, M. 2000, A\&A, 354, 135

Yonekura, Y., Dobashi, K., Mizuno, A., Ogawa, H., \& Fukui, Y. 1997, ApJS, 110,21

Zuckerman, B., Kim, S. S., \& Liu, T. 1995, ApJ, L79 
C. B. Pereira and L. F. Miranda: New post-AGB stars, Online Material $p 1$

\section{Online Material}




\section{Appendix A: PM 1-212, a young stellar object}

The spectrum of PM 1-212 presents the Balmer and the Paschen lines in absorption (Fig. 6). The strength of the Paschen line at $8751 \AA(8.4 \AA)$ is similar to the values found for A-type main sequence stars, after a comparison with stars from the sample of Danks \& Dennefeld (1994). In addition, the presence of the K line of Ca II at $3933 \AA$ further suggests an A-type star. In fact, inspecting the spectra of A-type stars from Jacoby et al. (1984), the presence of the $\mathrm{K}$ line and the absence of the $\mathrm{H}$ line also point to an A-type star. This happens because the Balmer lines attain their great strength at the early A-type stars. In this case, only the $\mathrm{K}$ line of the calcium doublet is seen, whereas the $\mathrm{H}$ line of calcium is severely blended with $\mathrm{H} \epsilon$ at $3970 \AA$. No helium lines were found in the spectrum of PM 1-212.

\section{Appendix B: H II regions}

\subsection{GLMP 1000}

The $\mathrm{H} \alpha$ and [N II] images (Fig. B.1) show three nebulous objects in the field, denoted A, B, and C, that are not detected in [O III]. Object A coincides with the position of GLMP 1000 indicated in SIMBAD. It extends $\sim 56^{\prime \prime}$ near the east-west direction, and morphologically can be described as a ring-like structure with extended "conical" emission toward the east. Its north-western edge is particularly bright. Object B is located close to the star GSC 03157-00393, presents a bow-shock like structure of 30" in size elongated along the east-west direction and is bright toward the north-west. Object $\mathrm{C}$ is a faint, compact nebulosity of $\sim 12^{\prime \prime}$ in size, although slightly elongated east-west. The intensity maximum of object $\mathrm{C}$ is also observed toward the west.

The spectrograph slit was centred on object A. The spectrum (Fig. B.2) shows nebular $\mathrm{H} \alpha$, [N II]6548,6583, [S II]6716,6731, and [S III]6312,9069 emission lines. The $\mathrm{H} \alpha$ emission extends $\sim 14^{\prime \prime}$, although diffuse $\mathrm{H} \alpha$ emission can be traced over $50^{\prime \prime}$. The other emission lines are detected over a spatial extent of $\sim 5^{\prime \prime}$. The $\mathrm{H} \alpha /[\mathrm{N}$ II $]$ and $\mathrm{H} \alpha /[\mathrm{S} \mathrm{II}]$ line intensity ratios are $\simeq 2.2$ and $\simeq 4.8$, respectively. These ratios are more compatible with object A being a photoionised region than a shock-excited nebulosity (Sabbadin et al. 1977).

These three objects are located near the bright star HD 196241 in the Cygnus OB2 association. Comerón et al. (2002) found an infrared star [CRP2002] B2 near the centre of the ring in object $\mathrm{A}$, which could be the exciting source of this object. It is clear that these three objects are related young stellar objects and not that are very evolved objects.

\subsection{GLMP 1062}

GLMP 1062 is located in a complex emission region (Fig. B.1). In addition to many emission and dark patches in the field, the most relevant structure is a large and knotty emission bow detected in $\mathrm{H} \alpha$ and [N II], but not in [O III]. GLMP 1062 is located near the "focus" of the bow, suggesting a physical relationship between the star and this structure. GLMP 1062 itself appears embedded in a faint nebulosity of $\simeq 10^{\prime \prime}$ in size detected in all three filters. The spectrum (Fig. B.2) shows nebular $\mathrm{H} \beta$, $\mathrm{H} \alpha$, [N II]6548,6583, [S II]6716,6731, and the permitted O I8446 emission lines. The $\mathrm{H} \alpha /[\mathrm{N}$ II $]$ and $\mathrm{H} \alpha /[\mathrm{S}$ II $]$ line intensity ratios are $\simeq 2.0$ and $\simeq 3.4$, respectively, indicating that GLMP 1062 is an $\mathrm{H}$ II region, although a contribution from shock-excitation cannot be ruled out. In our spectrum no emission from the bow has been detected, probably due to its faintness.

\section{Appendix C: GLMP 9, a G giant with infrared emission}

GLMP 9 is observed toward the young open cluster NGC 225, although it is not a member of the cluster (Lattanzi et al. 1991). In the direct images (not shown here), GLMP 9 appears stellar without traces of extended emission in the observed field. The object is suspected to be a T Tauri star by GL97 based on its IRAS colours. Figure 6 shows the spectrum of GLMP 9, which shows characteristics of a $\mathrm{G}$ star.

To determine its luminosity class, we have compared the measurements of the calcium infrared triplet with the stars of Danks \& Dennefeld (1994). The most straightforward conclusion is that GLMP 9 is not a supergiant star. As mentioned above, the strength of the calcium infrared triplet is strongest in supergiant stars. The strength of these lines in GLMP 9 is $1.5 \AA, 3.4 \AA$, and $2.9 \AA$ for the $8498 \AA, 8542 \AA$, and $8662 \AA$ lines, respectively. With these values, GLMP 9 lies in the region occupied by the giant stars in the $W(\mathrm{CaT}) \log \mathrm{g}$ relation of Jones et al. (1984). Another indication that GLMP 9 is a giant star is provided by the strength of the CN band around $7925 \AA$. In GLMP 9 the strength of this band is $2.3 \AA$. This value is in the range of the giant stars (Sect. 4.1.1). Moreover, among the G-type stars, this band is present in HD 20894 (giant), but is absent (or at least very weak) in HD 20766 (dwarf) (see Fig. 1 in Jones et al. 1984). Finally, in Fig. 7 we compare the spectrum of GLMP 9 with the spectrum of (HD 249240), a G7III star, and with that of (HD 22193), a G6V star reddened by $E(B-V)=0.5$, from the library spectra of Jacoby et al. (1984). We see that there is a good match between the spectrum of GLMP 9 and the spectrum of a giant star. Being a giant with infrared emission (GL97), GLMP 9 deserves further investigation. Several authors have tried to explain why stars with luminosity class III have infrared emission and as such several possibilities have been proposed (Zuckerman et al. 1995; Jura 1999).

\section{Appendix D: Objects with unclear classification}

\subsection{GLMP 75}

GLMP 75 is suspected to be T Tauri star by GL97 based on their IRAS colours. Our spectrum (Fig. D.1) shows that it has a reddened G-type spectrum. A tentative comparison was made using the library spectra of Jacoby et al. (1984) and we concluded that GLMP 75 is probably a dwarf. If GLMP 75 is a dwarf rather than a giant or a supergiant star with infrared emission, then it is a young stellar object.

\subsection{GLMP 1043}

GLMP 1043 is the faintest star of our sample from which we detect a continuum, albeit very noised (Fig. D.1). Based on the strength of the calcium infrared triplet, it seems that GLMP 1043 is a late G-early $\mathrm{K}$ star main sequence star, although this should be seen with caution because of the uncertainty of the continnum in this region.

\subsection{GLMP 1083}

Classified by GL97 as a T Tauri star, our spectrum shows a very reddened star, with $\mathrm{H} \alpha$, the oxygen triplet at $7774 \AA$, O I8446 $\AA$, and the calcium infrared triplet in emission (Fig. D.1). As already mentioned in Sect. 4.3, the calcium infrared triplet in emission is usually a strong feature in the spectra of young stellar 
C. B. Pereira and L. F. Miranda: New post-AGB stars, Online Material $p 3$
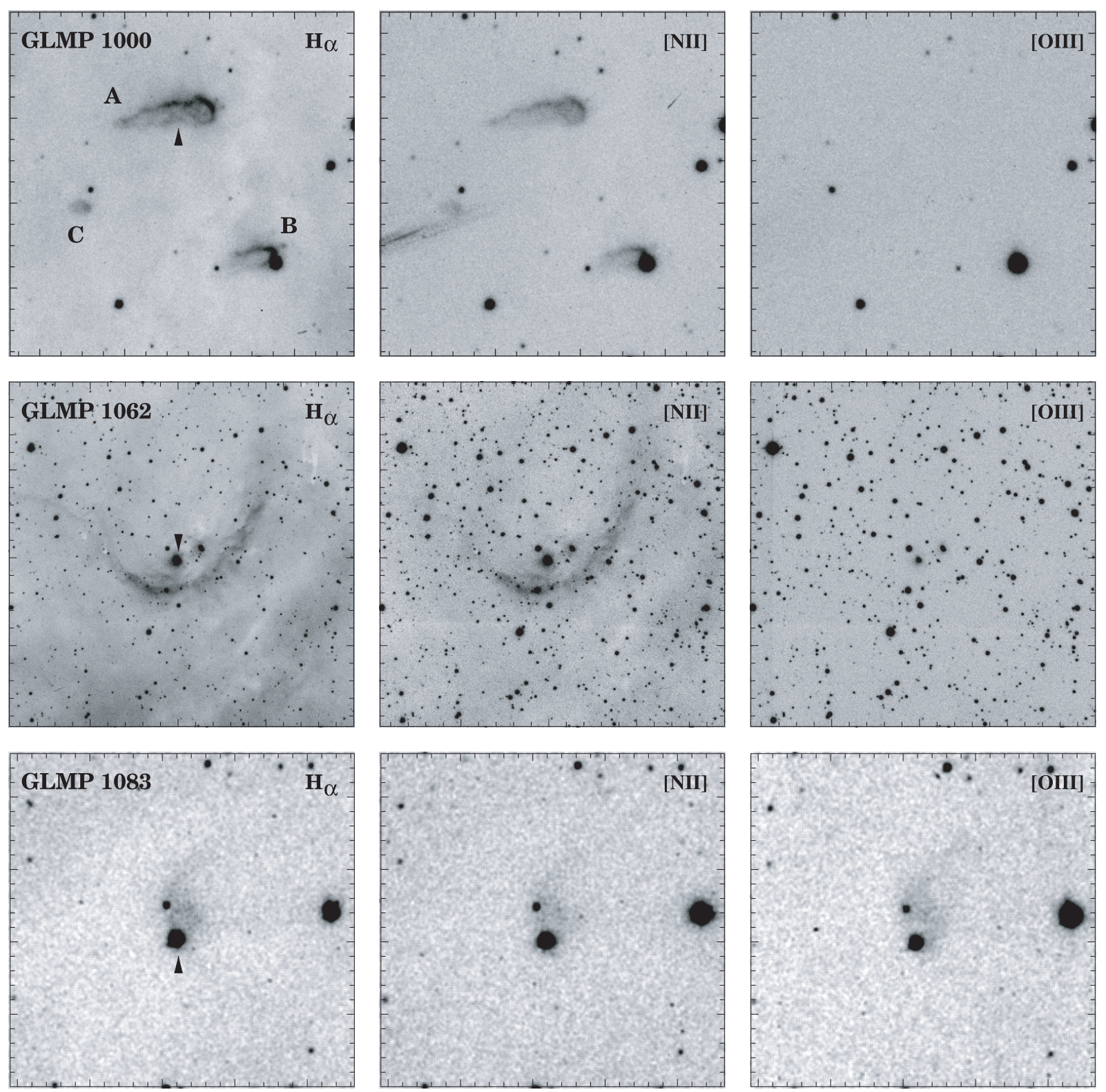

Fig. B.1. Grey-scale reproductions of the $\mathrm{H} \alpha$, [N II], and [O III] images of GLMP 1000, GLMP 1062, and GLMP 1083. The grey levels are linear. North is up, east to the left. The size of the field shown is 3'.1 × 3'.1 in GLMP 1000, 7'.5 × 7'.5 in GLMP 1062, and 1'.8 × 1.76 in GLMP 1083. The arrow in the $\mathrm{H} \alpha$ frames indicates the object classified as candidate to post-AGB according to SIMBAD. The three nebulous objects observed in GLMP 1000 have been denoted A, B, and C. The linear emission features observed in the [N II] image of GLMP 1000 eastwards of object C are an artifact.

objects, suggesting that GLMP 1083 is a young stellar object. However, since it is also present in GLMP 1015, the probability of GLMP 1083 being a post-AGB star cannot be disregarded.

GLMP 1083 is observed toward the dark nebula LDN 1253 in the Cep OB3 association (see Yonekura et al. 1997), although from our data we cannot conclude whether it belongs to LDN 1253. In our images (Fig. B.1), the object seems to be associated with nebulosity that presents the shape of a cometary nebula extending $\simeq 7^{\prime \prime}$ northwards from the star. This nebula has already been identified by Magakian (2003) as the reflection nebula GN 23.43.6. However, the coordinates provided by Magakyan correspond to the faint star observed $\simeq 10^{\prime \prime}$ north of GLMP 1083. From our images we cannot decide which object is the illuminating star of GN 23.43.6. 
C. B. Pereira and L. F. Miranda: New post-AGB stars, Online Material p 4

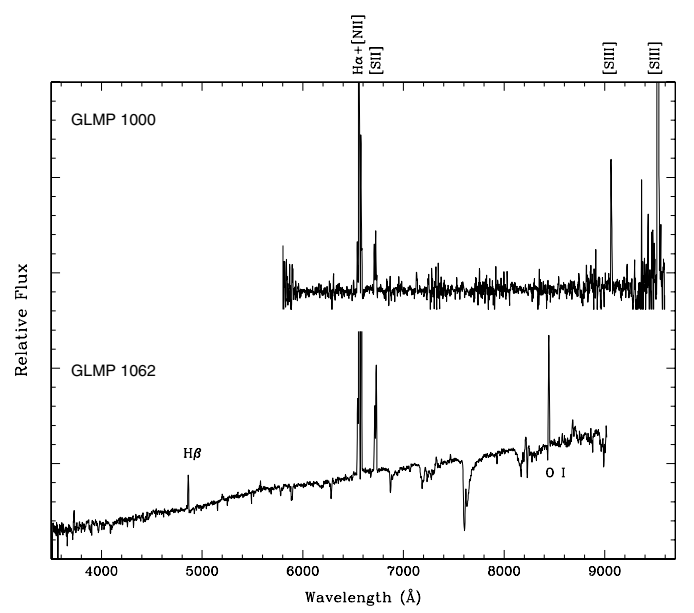

Fig. B.2. Relative flux versus wavelength $(\AA)$ spectra of GLMP 1000 and GLMP 1062.

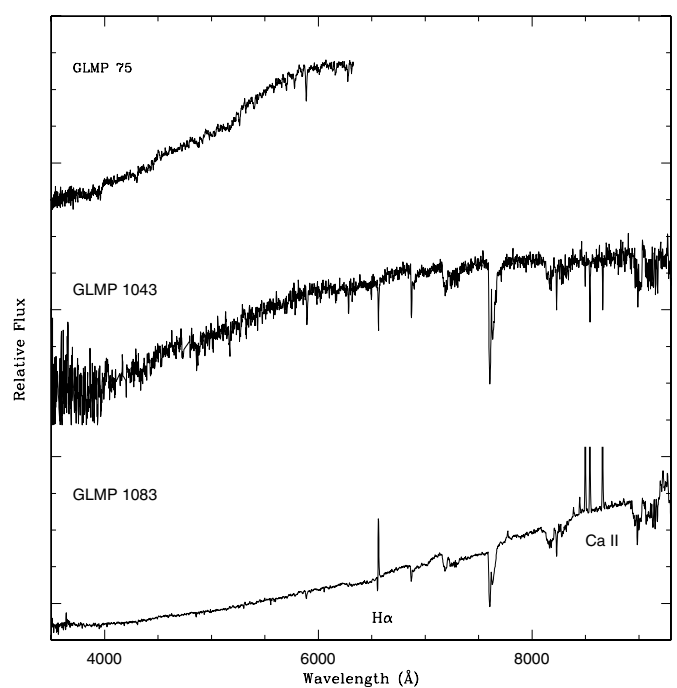

Fig. D.1. Relative flux versus wavelength ( $\AA$ ) spectra of GLMP 75, GLMP 1043, and GLMP 1083. 